\title{
EDITORIAL
}

For reprint orders, please contact: reprints@futuremedicine.com

\section{Adjuvant hormonal therapy in young breast cancer patients}

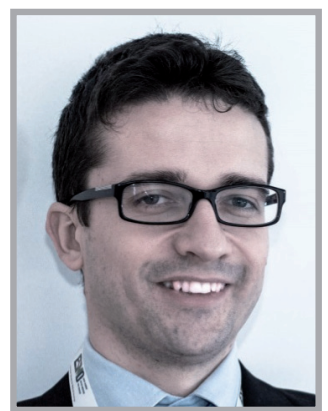

Matteo Lambertini ${ }^{1}$

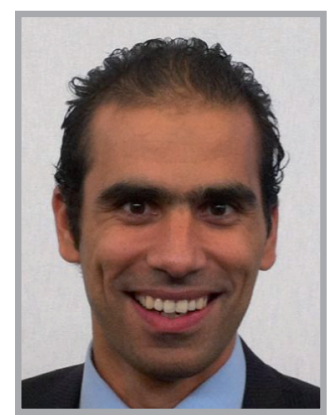

Hatem A Azim Jr*2
"Approximately 5\% of
women with breast cancer are diagnosed before the age of 40 years, but the incidence appears to be on the rise."
Breast cancer arising at a young age is a complex condition, requiring multidisciplinary and personalized approaches to manage the specific issues related not only to optimal adjuvant treatments, but also to other important quality-of-life issues, such as bone health, premature menopause, fertility preservation and long-term cognitive function [1]. Approximately $5 \%$ of women with breast cancer are diagnosed before the age of 40 years, but the incidence appears to be on the rise [101].

In the adjuvant setting, 5 years of tamoxifen treatment is considered the current standard of care in managing young women with an endocrine-sensitive disease. Based on the meta-analysis of the Early Breast Cancer Trialists' Collaborative Group (EBCTCG), 5 years of tamoxifen significantly reduced breast cancer mortality throughout the first 15 years, with a greater effect when tamoxifen was given for 5 years compared with 2 years [2]. Nevertheless, in the past decade several questions were raised and remain unanswered in the field of adjuvant endocrine treatment of young breast cancer patients, including the optimal duration of adjuvant endocrine therapy, the addition of luteinizing hormone-releasing hormone analogs (LHRHa) to standard tamoxifen and its duration, and the use of aromatase inhibitors.

The first evidence regarding the possible benefit of extending adjuvant endocrine therapy beyond 5 years of tamoxifen was based on the results of the MA.17 trial, where patients were randomized to receive or not receive letrozole following the completion of tamoxifen treatment. The main analysis indicated that women originally assigned to receive letrozole had a $32 \%$ reduction in the risk of relapse [3]. A subgroup analysis suggested that the benefit in terms of disease-free survival was greater in premenopausal women at the time of initial diagnosis than in postmenopausal patients [3]. More recently, the results of two important clinical trials that assessed the possible benefit of extending adjuvant tamoxifen up to 10 years instead of stopping it after 5 years became available $[4,5]$. The ATLAS trial included a total of 12,894 patients: 2482 (19\%) were younger than 45 years at diagnosis and 1058 (8\%) were known to be premenopausal at the

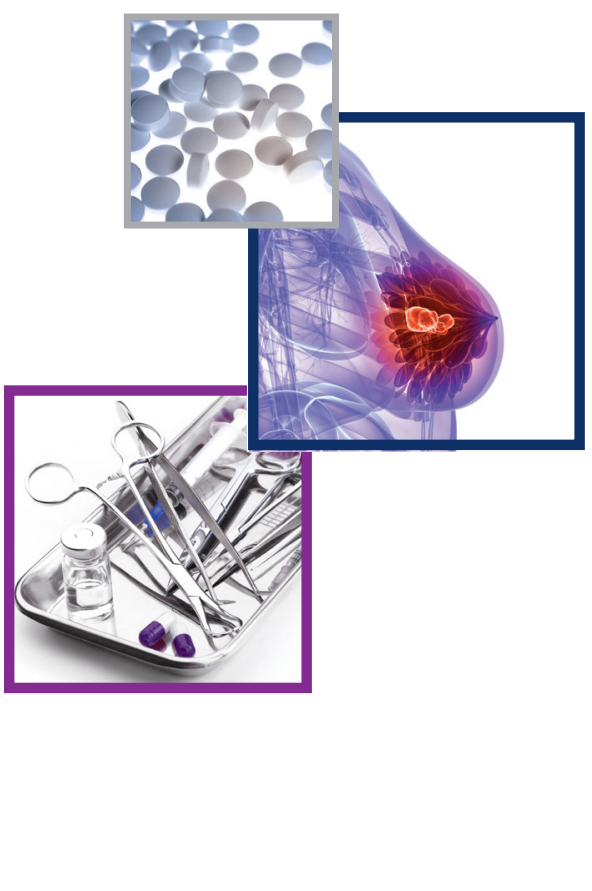

\footnotetext{
“...in the past decade several

questions were raised and remain unanswered in the field of adjuvant endocrine treatment of young breast cancer patients..."
} 
"...challenges exist in deciding to use the optimal adjuvant endocrine treatment, including long-term side effects and fertility issues, with both having a considerable effect on the adherence of young women to endocrine treatment." time of study entry [4]. Patients were randomized after the completion of 5 years of tamoxifen treatment to either 5 more years or observation. At a mean follow-up of 7.6 years, the continuation of tamoxifen up to 10 years led to a statistically significant reduction in the risk of recurrence, breast cancer mortality and overall mortality $(\mathrm{p}=0.002, \mathrm{p}=0.01$ and $\mathrm{p}=0.01$, respectively) [4]. Mortality without recurrence from causes other than breast cancer was as low as $1 \%$ [4]. Similar results were shown by another study (aTTom), which included a total of 6953 patients [5]. Importantly, the benefit appears to be observed similarly both in pre- and post-menopausal patients. Acknowledging that aromatase inhibitors are becoming the standard of care for postmenopausal patients, the results of ATLAS and aTTom appear to be of higher relevance for young breast cancer patients. Recently, a large, prospective study showed a steady increase over time in the risk of relapse for patients with estrogen receptor-positive disease [6]. This observation further emphasizes the validity of considering extending the period of adjuvant hormonal therapy beyond 5 years, particularly in patients with a high absolute risk of recurrence (e.g., node-positive disease).

To date, no large, randomized studies have investigated the added value of LHRHa to tamoxifen. In the meta-analysis by Cuzick and colleagues, the addition of an LHRHa to tamoxifen versus tamoxifen alone (data available for 1013 patients) did not demonstrate a significant impact on the risk of recurrence or the risk of death [7]. The results of the SOFT trial [102] are eagerly awaited to understand the potential added value of ovarian function suppression to tamoxifen compared with tamoxifen alone. This trial is expected to report in 2014.

Aromatase inhibitors alone are contraindicated in premenopausal women. Premenopausal patients who received letrozole plus LHRHa for at least 6 months demonstrated a more profound suppression of median estradiol serum levels and luteinizing hormone levels, and showed higher median follicle-stimulating hormone serum levels than patients who received tamoxifen plus LHRHa [8]. Moreover, the neoadjuvant administration of anastrozole plus goserelin in premenopausal patients produced a superior overall tumor response compared with tamoxifen plus goserelin [9]. These findings supported the hypothesis that aromatase inhibitors could be more active than tamoxifen as an adjuvant endocrine therapy for premenopausal breast cancer patients. This hypothesis has been tested in the ABCSG-12 trial, which evaluated the efficacy of 3 years of treatment with LHRHa plus anastrozole or tamoxifen with or without zoledronic acid in 1803 premenopausal women with early low-risk breast cancer [10]. However, at a median follow-up of 62 months, no difference in disease-free survival was observed between both arms, with a worse overall survival observed in the anastrozole arm [10]. Currently, two studies including more than 5000 patients are addressing the same question (SOFT [102] and TEXT [103]), and their results are expected in 2014. Until then, ovarian suppression in combination with an aromatase inhibitor should not be considered unless in patients with a contraindication to tamoxifen [11].

Apart from the aforementioned issues, other challenges exist in deciding to use the optimal adjuvant endocrine treatment, including longterm side effects and fertility issues, with both having a considerable effect on the adherence of young women to endocrine treatment. A recent meta-analysis showed that adherence to adjuvant endocrine therapy is suboptimal in young breast cancer patients [12]. As shown in the ELIPPSE 40 cohort study, the percentage of breast cancer patients younger than 40 years of age who discontinued tamoxifen amounted to $40 \%$ after 3 years of endocrine therapy [13]. Indeed, interventions such as providing information systematically and repeatedly about the importance of therapy are required to improve their compliance.

Serious considerations should be given to the development of side effects that can compromise quality of life of breast cancer survivors. Young women receiving endocrine treatment are at high risk for developing menopausal-type symptoms, including hot flushes, early awakening, forgetfulness, breast sensitivity, weight gain, vaginal dryness and night sweats [14]. Particularly, women receiving ovarian suppression therapy were shown to be the most likely to have moderate or severe menopausal-type symptoms [14]. These findings should be taken into account when discussing with young breast cancer patients the risks and benefits of the available endocrine therapies. Pharmacological therapies, behavioral therapies, complementary medicine and alternative medicine approaches are available for some symptoms, but further research is needed in this field to ameliorate quality of life of breast cancer survivors. 
Issues related to fertility and pregnancy should be addressed, particularly in patients who did not complete their family plans before breast cancer diagnosis $[15,16]$. Fertility preservation options should be discussed as early as possible before commencing treatment. This is particularly important for patients who are candidates to extended endocrine therapy, for whom the diagnosis of breast cancer could negatively impact their desire to have children. On this regard, a prospective study carried on by the Breast International Group and North American Breast Cancer Group (BIG-NABCG) is going to start [17]. The trial is being carried out in young women with endocrine-sensitive early breast cancer who desire to become pregnant and who are disease free after 2 years of adjuvant endocrine therapy, with the aim to evaluate the feasibility and safety of a temporary treatment interruption to allow conception [17].

Finally, it should not be forgotten that breast cancer in young women has a worse prognosis than older patients, with a particular trend observed in patients with ER-positive disease, especially with luminal-B tumors, which appear to be more common in younger patients $[18,19]$. These findings highlight the need for new treatment approaches. Recent analyses using gene-expression profiling showed that breast cancer in the young has a unique biology and is enriched with genes involved in growth factor signaling (e.g., PI3K signaling) [18,20]. PI3K is the most frequently altered pathway in breast cancer and plays a pivotal role in endocrine resistance [20]. This strategy is currently being tested in the metastatic setting and could emerge as being of particular interest in younger patients where the pathway appears to be highly activated.

In conclusion, owing to the lack of studies addressing many of the several issues related to the treatment of breast cancer in young women, future research in this domain should be considered a priority. Given the relative rarity of the disease, international collaborations are needed to help further research development in this field.

\section{Financial \& competing interests disclosure}

$M$ Lambertini is supported by an ESMO Clinical Unit Visit grant. The authors have no other relevant affiliations or financial involvement with any organization or entity with a financial interest in or financial conflict with the subject matter or materials discussed in the manuscript apart from those disclosed.

No writing assistance was utilized in the production of this manuscript.

\section{References}

1 Cardoso F, Loibl S, Pagani O et al. The European Society of Breast Cancer Specialists recommendations for the management of young women with breast cancer. Eur. J. Cancer 48, 3355-3377 (2012).

2 Early Breast Cancer Trialists' Collaborative Group (EBCTCG). Relevance of breast cancer hormone receptors and other factors to the efficacy of adjuvant tamoxifen: patient-level meta-analysis of randomized trials. Lancet 378, 771-784 (2011).

3 Higgins MJ, Liedke PE, Goss PE. Extended adjuvant endocrine therapy in hormone dependent breast cancer: the paradigm of the NCIC-CTGMA.17/BIG 1-97 trial. Crit. Rev. Oncol. Hematol. 86, 23-32 (2013).

4 Davies C, Pan H, Godwin R et al. Long-term effects of continuing adjuvant tamoxifen to 10 years versus stopping at 5 years after diagnosis of oestrogen receptorpositive breast cancer: ATLAS, a randomized trial. Lancet 381, 805-816 (2013).
5 Gray RG, Rea D, Handley K et al. aTTom: long-term effects of continuing adjuvant tamoxifen to 10 years versus stopping at 5 years in 6953 women with early breast cancer. J. Clin. Oncol. 31(Suppl.), Abstract 5 (2013).

6 Copson E, Eccles B, Maishman T et al. Prospective observational study of breast cancer treatment outcomes for UK women aged 18-40 years at diagnosis: the POSH study. J. Natl Cancer. Inst. 105, 978-988 (2013).

7 Cuzick J, Ambroisine L, Davidson N et al.; LHRH-agonists in Early Breast Cancer Overview group. Use of luteinisinghormone-releasing hormone agonists as adjuvant treatment in premenopausal patients with hormone-receptor-positive breast cancer: a meta-analysis of individual patient data from randomized adjuvant trials. Lancet 369, 1711-1723 (2007).

8 Rossi E, Morabito A, De Maio E et al. Endocrine effects of adjuvant letrozole + triptorelin compared with tamoxifen + triptorelin in premenopausal patients with early breast cancer. J. Clin. Oncol. 26, 264-270 (2008).

9 Iwata H, Masuda N, Sagara Y et al. Analysis of Ki-67 expression with neoadjuvant anastrozole or tamoxifen in patients receiving goserelin for premenopausal breast cancer. Cancer 119, 704-713 (2013).

10 Gnant M, Mlineritsch B, Stoeger H et al. Adjuvant endocrine therapy plus zoledronic acid in premenopausal women with early-stage breast cancer: 62-month follow-up from the ABCSG-12 randomised trial. Lancet Oncol. 12, 631-641 (2011).

11 Goldhirsch A, Winer EP, Coates AS et al. Personalizing the treatment of women with early breast cancer: highlight of the $\mathrm{St}$ Gallen International Expert Consensus on the Primary Therapy of Early Breast Cancer 2013. Ann. Oncol. 24(9), 2206-2223 (2013).

12 Murphy CC, Bartholomew LK, Carpentier MY, Bluethmann SM, Vernon SW. Adherence to adjuvant hormonal therapy among breast cancer survivors in clinical practice: a systematic review. Breast Cancer Res. Treat. 134, 459-478 (2012). 
13 Huiart L, Bouhnik AD, Rey D et al. Early discontinuation of tamoxifen intake in young women with breast cancer: is it time to rethink the way it is prescribed? Eur. J. Cancer 48, 1939-1946 (2012).

14 Leining MG, Gelber S, Rosenberg R, Przypyszny M, Winer EP, Partridge AH. Menopausal-type symptoms in young breast cancer survivors. Ann. Oncol. 17, 1777-1782 (2006).

15 Loren AW, Mangu PB, Beck LN et al. Fertility preservation for patients with cancer: American Society of Clinical Oncology clinical practice guideline update. J. Clin. Oncol. 31, 2500-2510 (2013).

16 Peccatori FA, Azim HA Jr, Orecchia R et al. Cancer, pregnancy and fertility: ESMO Clinical Practice Guidelines for diagnosis, treatment and follow-up.
Ann. Oncol. 24(Suppl. 6), vi160-vi170 (2013).

17 Pagani O, Azim H Jr. Pregnancy after breast cancer: myths and facts. Breast Care (Basel) 7 , 210-214 (2012).

18 Azim HA Jr, Michiels S, Bedard PL et al. Elucidating prognosis and biology of breast cancer arising in young women using gene expression profiling. Clin. Cancer Res. 18, 1341-1351 (2012).

19 Cancello G, Maisonneuve P, Rotmensz N et al. Prognosis and adjuvant treatment effects in selected breast cancer subtypes of very young women ( $<35$ years) with operable breast cancer. Ann. Oncol. 21, 1974-1981 (2010).

20 Anders CK, Acharya CR, Hsu DS et al. Age-specific differences in oncogenic pathway deregulation seen in human breast tumors. PLoS One 3, e1373 (2008).

\section{- Websites}

101 Surveillance, Epidemiology and End Results (SEER).

www.seer.cancer.gov

102 ClinicalTrials.gov. Suppression of Ovarian Function Plus Either Tamoxifen or Exemestane Compared With Tamoxifen Alone in Treating Premenopausal Women With Hormone-Responsive Breast Cancer (SOFT).

http://clinicaltrials.gov/ct2/show/ NCT00066690

103 ClinicalTrials.gov. Triptorelin With Either Exemestane or Tamoxifen in Treating Premenopausal Women With HormoneResponsive Breast Cancer (TEXT). http://clinicaltrials.gov/ct2/show/ NCT00066703 